\title{
Editorial: Recent Advances on the Mobile Multimedia Services and Applications
}

\author{
Shengping Zhang ${ }^{1} \cdot$ Dianhui Chu $^{1} \cdot$ Yanxiao Zhao ${ }^{2} \cdot$ Dalei $\mathrm{Wu}^{3} \cdot$ Qing Yang $^{4}$ \\ Published online: 20 November 2020 \\ (C) Springer Science+Business Media, LLC, part of Springer Nature 2020
}

Editorial:

The successful deployment of multimedia services and applications in a mobile environment requires an interdisciplinary approach, where multimedia, network and physical layer issues will be solved jointly. Content features analysis and coding, media access control, multimedia flow and error control, cross-layer optimization, Quality of Experience (QoE), media cloud as well as mobility management and security protocols are research challenges. These challenges need to be carefully checked when designing new mobile media architecture. A great effort is needed to be put into designing applications, taking into account users' perceptions of the overall quality of the services provided. Within this scope, MOBIMEDIA aims to provide a unique international forum for researchers from industry and academia, who are dedicated to the fields of multimedia coding, mobile communications and networks to study new technologies, applications and standards. The collection of original unpublished manuscripts can improve the knowledge and practice of the integrated design of efficient technologies and the provision of advanced mobile multimedia applications.

This special issue features five selected papers with high quality. The first article, "Detection of Fake Reviews using Group Model", proposed the concept of review group, which is designed to effectively split reviews of reviewer into groups to identify both positive and negative deceptive reviews. Additionally, authors explore the collusion relationship

Shengping Zhang

s.zhang@hit.edu.cn

1 Harbin Institute of Technology, 92 Xidazhi St, Nangang, Harbin, Heilongjiang, China

2 Virginia Commonwealth University, 907 Floyd Ave, Richmond, VA 23284, USA

3 The University of Tennessee at Chattanooga, 615 McCallie Ave, Chattanooga, TN 37403, USA

4 University of North Texas, 1155 Union Cir, Denton, TX 76203, USA between reviewers to build reviewer group collusion model. The algorithms can effectively improve the precision in fake reviews classification task especially when reviews are posted by professional review spammers.

With the development of neural network models, how to compress models and accelerate neural networks are undoubtedly to be crucial research topic. The second article titled "BitQuantized-Net: An Effective Method for Compressing Deep Neural Networks" studied the problem that neural network models suffer from computational consuming and memory intensive for parameters training/storage. Author proposed "Bit-Quantized-Net"(BQ-Net), which trains the network with bit quantized weight to shorten the running time and applies Huffman coding to compressed the model size.

In the next article with the title "Node Attitude Aware Information Dissemination Model Based on Evolutionary Game in Social Networks", the authors explored the influence of node attitude on information dissemination and proposed an information propagation model based on evolutionary game. By considering both node's attitude update rules and inter-node game matrix, both positive and negative postures replicate the equilibrium solutions of the dynamic equations and stabilize the corresponding equilibrium points. This model show that the different attitudes of nodes play an important role in information dissemination.

Effective feature representation is widely regarded as the most important premise for sensor-based activity recognition (AR). The fourth article titled "Enhancing Representation of Deep Features for Sensor-based Activity Recognition" improved the feature representation of activities by using a reversed $\mathrm{CNN}$ to generate the significant data based on the original features and combine the raw training data with significant data to obtain enhanced training data. This algorithm can not only train better feature extractors but also help better understand the abstract features of sensor-based activity data.

The last article titled "Indoor WiFi Positioning Algorithm Based on Location Fingerprint", authored by Chunshan Li, presented a method based on skewness-kurtosis normality test and Kalman filter fusion through the research of fingerprint 
data. Based on the traditional Kalman filter method, the proposed method optimizes the three stages of the fingerprint positioning algorithm through the skewness-kurtosis normality, the weighted KNN (K-Nearest Neighbor), the fusion of Levenberg-Marquardt method and the Kalman filter to improve the accuracy of positioning.

Acknowledgements The guest editors are thankful to our reviewers for their effort in reviewing the manuscripts. We also thank the Edit-in-Chief, Dr. Imrich Chlamtac for his supportive guidance during the entire process.

Publisher's note Springer Nature remains neutral with regard to jurisdictional claims in published maps and institutional affiliations.

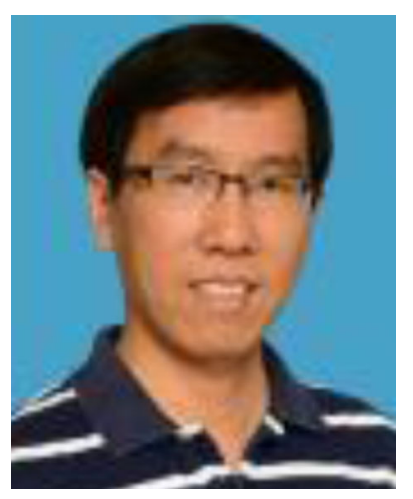

Shengping Zhang received the $\mathrm{Ph} . \mathrm{D}$. degree in computer science from the Harbin Institute of Technology, Harbin, China, in 2013. He is currently a Professor with the School of Computer Science and Technology, Harbin Institute of Technology, Weihai. He had been a Postdoctoral Research Associate with Brown University and Hong Kong Baptist University, and a Visiting Student Researcher with the University of California at Berkeley. He has authored or coauthored over 60 research publications in refereed journals and conferences. His research interests include deep learning and its applications in computer vision.

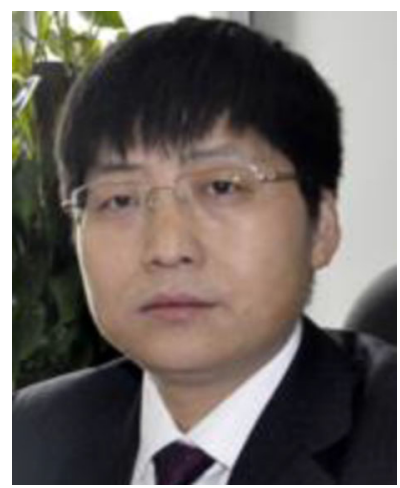

Dianhui Chu received the Ph.D. degree from the Harbin Institute of Technology, Harbin, China, in 2014. He is currently a Professor with the School of Computer Science and Technology, Harbin Institute of Technology at Weihai. His research interests are concentrated on fuzzy systems and knowledge discovery.

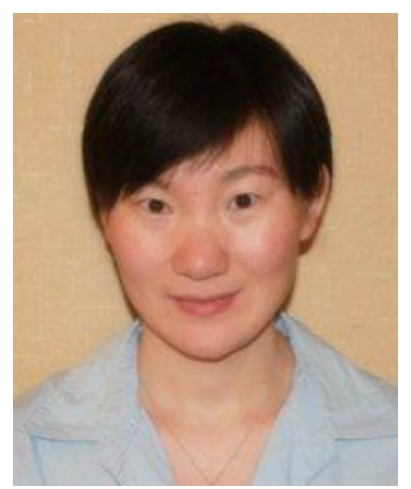

Yanxiao Zhao is an Associate Professor in the Electrical and Computer Engineering De partment, Virginia Commonwealth University, where she joined in Aug. 2018. Prior to joining VCU, Dr. Zhao was a faculty member at South Dakota School of Mines and Technology from 2012 to 2018. She received her Ph.D. degree from the Department of Electrical and Computer Engineering, Old Dominion University, USA, in May 2012, under the supervision of Dr. Min Song. Dr. Zhao's research interests include, but not limited to: cyber security, wireless networks including vehicular networks, wireless autonomous networks, wireless body area networks, software-defined networks, and device-to-device (D2D) communications, energy harvesting for Internet of things with wearable sensors, power management and communications in smart grid. Dr. Zhao's research has been supported by NSF, NASA and Air Force. Dr. Zhao has published over 70 papers in prestigious journals and international conferences. She was the recipient of the Best Paper Award for three international conferences WASA2009, ChinaCom2016 and ICMIC2019. She has been actively organizing international conferences by serving as TPC co-chair, publicity chairs and TPC members. She is an IEEE Senior Member.

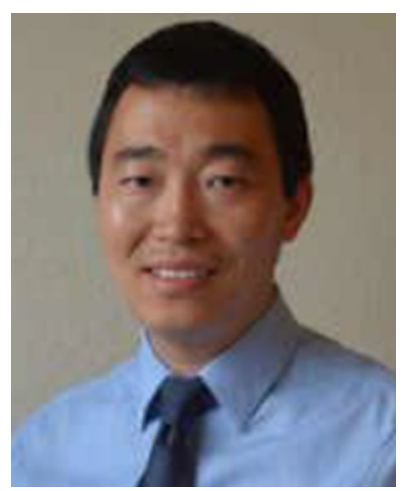

Dalei $\mathbf{W u}$ is a UC Foundation Assistant Professor with the Department of Computer Science and Engineering at the University of Tennessee at Chattanooga (UTC). Before joining UTC, he worked as a Postdoctoral Researcher with the Mechatronics Research Laboratory at Massachusetts Institute of Technology (MIT). His areas of expertise include cyber-physical systems, intelligent systems, multimedia computing, and mobile computing. His is particularly interested in using methods of modeling, simulation, optimization, and machine learning to solve real-world problems. He has published research papers in some prestigious journals, such as, ACM Transactions on Modeling and Computer Simulation, IEEE Transactions on Industrial Informatics, IEEE Transactions on Automatic Control, IEEE Transactions on Circuits and Systems for Video Technology, IEEE Transactions on Wireless Communications, IEEE Journal on Selected Areas in Communications, IEEE Transactions on Multimedia, and IEEE Transactions on Communications. His research has been funded by NSF and the state of Tennessee. 


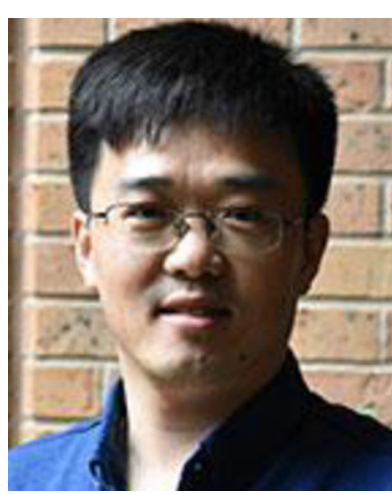

Qing Yang is an assistant professor in the Department of Computer Science and Engineering at University of North Texas, TX. He received his $\mathrm{Ph} . \mathrm{D}$ degree in Computer Science and Software Engineering from Auburn University, AL, USA in 2011. His current research interests include connected and autonomous vehicles, Internet of Things, network security and privacy. His research is funded by the U.S. National Science Foundation, U.S. Federal Highway Administration, Office of Naval Research,
Toyota InfoTech Inc., Fujitsu Laboratories of America Inc., and the University of North Texas Office of the Vice President for Research and Innovation. 